\title{
GENETICS
}

\section{Allelic drive in Drosophila}

Guichard, A. et al. Nat. commun. 10: 1640 (2019)

Gene drive systems circumvent the rules of Mendelian inheritance by increasing the probability that a DNA sequence will be transmitted to offspring to over $50 \%$, thereby resulting in the faster propagation of the DNA sequence within a population across generations. Scientists have long recognized the potential of developing synthetic gene drive strategies for basic and applied research, with potential applications that include modifying insect populations to reverse pesticide resistance and control invasive species. Recent studies have reported the efficient super-Mendelian inheritance of transgenic insertional elements in insects and mice via CRISPR/ Cas9 gene editing-mediated gene drive approaches; a new study in Drosophila takes gene drive a step further by describing an allelic drive process that manipulates the inheritance of allelic variants.

The investigators reasoned that in heterozygous individuals carrying two different alleles of a gene, CRISPR/Cas9 gene editing could repair a cleavage-sensitive allele with a cut-resistant allele of the same gene provided in trans. Noticing that an Abruptex mutation $\left(N^{A x 16}\right)$ in the X-linked Drosophila Notch $(N)$ locus eliminates a PAM site in the wild-type $N^{+}$allele, the investigators designed a guide RNA (gRNA) anchored to this PAM site to direct cleavage of the wild-type $N^{+}$allele but not of the $N^{A x 16}$ allele. The guide was included in a CopyCat $(\mathrm{ccN})$ element designed to insert and copy itself into a yellow $(y)$ locus in the X chromosome. The investigators hypothesized that in the presence of Cas9, the dual gRNA drive system should result in the super-Mendelian inheritance of both the gene drive element $\left(y^{c c N}\right)$ and the $N^{A x 16}$ allele via copy-cutting. F0 females carrying the $y^{c c N}$ and $N^{A x 16}$ alleles were crossed to males homozygous for Cas9 to generate F1 $y^{c c N}$ $N^{A x 16} /++$; Cas9/+ female progeny, and F1 females were then crossed to wild-type male $\left(y^{+} N^{+}\right)$. Analysis of F2 progeny confirmed the highly biased inheritance of both $y^{c c N}$ and $N^{A x 16}$ alleles, thereby confirming the efficiency of the allelic drive. A similar efficiency was observed when allelic drive was achieved through copy-grafting, a more general strategy in which the favored allele is associated with neighboring sequences resistant to gRNA cleavage.

The study also characterizes two phenomena that contribute to the efficient allelic drive observed. Lethal mosaicism and shadow drive are mediated by maternally perduring Cas9-gRNA complexes and are respectively responsible for the elimination of the $\mathrm{N}^{-}$allele generated by imprecise DNA repair mediated by the non-homologous end-joining (NHEJ) pathway and for the residual drive observed in F3 generation. "In animal models, allelic-drives could accelerate the construction of complex genotypes for biomedical and basic research," conclude the investigators.

Alexandra Le Bras

Published online: 21 May 2019

https://doi.org/10.1038/s41684-019-0317-7

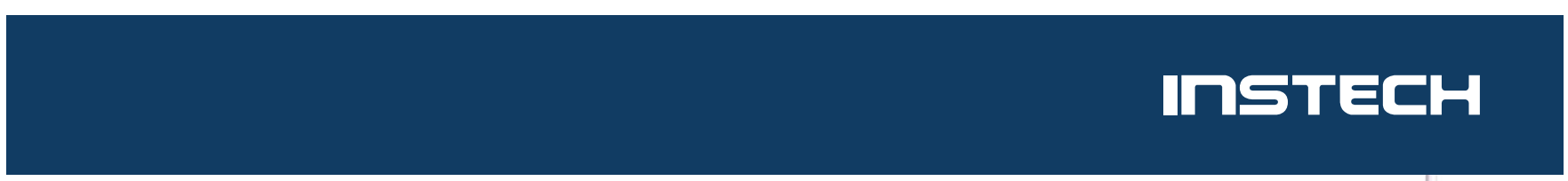

\section{Oral gavage, refined.}

Our plastic feeding needles have flexible tubes with soft tips to reduce trauma, and they're disposable to eliminate the hassle of cleaning.

Available in a range of sizes for mice, rats and other rodents. www.instechlabs.com
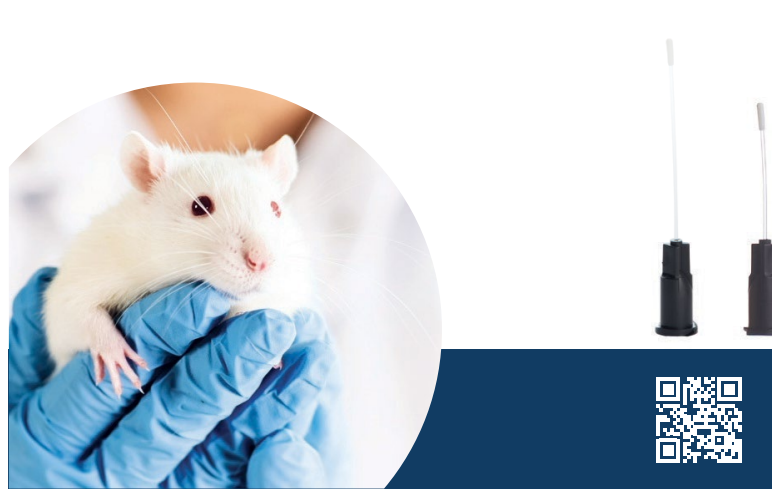